\title{
Uso de medios electrónicos como estrategia de enseñanza para generar un aprendizaje dinámico e interactivo
}

\author{
Use of electronic media as a teaching strategy to generate a dynamic and interactive learning. \\ Hugo Alarcon-Acosta ${ }^{a}$, Luz Eloisa Mendoza-Hernández $^{b}$, Luz Arely Monroy-González $^{c}$
}

\begin{abstract}
:
Currently there are various types of electronic education, which can provide benefits depending on the sector to which they want to apply. Based on this statement, we consider that it is important to know what type of learning is the one that students prefer this in order to obtain better results in the learning process. To achieve this purpose, a survey was applied to a representative sample of students who attend the second semester of high school (baccalaureate), which showed results about the importance that students give to electronic media as tools for dynamic and interactive learning. To address the issue, in this research report we present part of these results and prior to that, we address the relevance of the topic at present and we show a conceptual review about the types of electronic learning, which also served as the basis for the derivation of conclusions.
\end{abstract}

Keywords:

E-learning. Types of e-learning. Learning styles. M-Learning

\section{Resumen:}

Actualmente existen diversos tipos de enseñanza electrónica, que pueden brindar beneficios dependiendo del sector al que se quieran aplicar. Tomando como base esta afirmación consideramos que es importante conocer qué tipo de aprendizaje es el que los estudiantes prefieren esto con la finalidad de obtener mejores resultados en el proceso de aprendizaje. Para alcanzar este propósito se aplicó una encuesta a una muestra representativa de estudiantes que cursan el segundo semestre de la educación preparatoria (bachillerato), la cual arrojó resultados sobre la importancia que dan los estudiantes a los medios electrónicos como herramientas para el aprendizaje dinámico e interactivo. Para abordar la temática, en este reporte de investigación presentamos parte de dichos resultados y previo a ello, abordamos la relevancia del tema en la actualidad y mostramos una revisión conceptual sobre los tipos de aprendizaje electrónico, lo que también sirvió como base para la derivación de conclusiones.

\section{Palabras Clave:}

Aprendizaje electrónico, Tipos de aprendizaje electrónico, Estilos de aprendizaje, Aprendizaje móvil

\section{Introducción}

La aplicación de las Tecnologías de la Información y de la Comunicación (TIC), y en particular del uso de Internet dentro del ámbito educativo en la actualidad representan un gran aliado en el día a día, porque nos ofrecen un gran número de recursos, los cuales nos permiten la transmisión y generación del conocimiento, de esta manera se tiene un acceso universal por lado a la información en general y por otro, el poder establecer comunicación para intercambiar información y experiencias, en donde existe una igualdad en la instrucción; bachilleres de diversas partes del país, incluso del mundo.

Dentro del proceso de la enseñanza y el aprendizaje que toma como referente la web brinda la posibilidad de ofrecer recursos actualizados e ilimitados, lo que permite proponer material creativo; así las tecnologías de la información y comunicación, han brindado diversos beneficios a la sociedad, entre éstas se pueden destacar aquellos brindados a la educación (De Benito, 2000).

Hoy en día estamos llenos de nuevas herramientas tecnológicas que ayudan a reducir la brecha educativa, al brindar acceso a la información de cualquier parte del mundo. Tomando en cuenta estos planteamientos, se puede destacar que el aprendizaje también se ha visto influido por este tipo de innovaciones. Por ello, han surgido diferentes tipos de aprendizaje electrónico como; e-Learning, b-Learning, m-Lerarnign y u-Learning (Pérez, M., \& Delgado, A.

El aprendizaje electrónico o también conocido como elearning, hace referencia a una educación a distancia,

\footnotetext{
a Profesor de la Escuela Preparatoria No. 1, UAEH, Pachuca de Soto, Hidalgo: México, Email: hugo_alarcon@uaeh.edu.mx

${ }^{\mathrm{b}}$ Profesora de la Licenciatura en Ciencias de la Educación, UAEH, Pachuca de Soto, Hidalgo: México, Email: eloisamh@uaeh.edu.mx

c Profesora de la Escuela Preparatoria No. 1, UAEH, Pachuca de Soto, Hidalgo: México, Email: luz_monroy10358@uaeh.edu.mx
} 
utilizando los diferentes canales electrónicos que nos brindan las TIC, como las aplicaciones de hipertexto, entre las que encontramos: el correo electrónico, páginas web, foros de discusión, mensajería instantánea, plataformas de formación, redes sociales, etc. Pero no solo a través de los estándares tecnológicos se puede llevar a cabo este tipo de aprendizaje, ya que también se puede hacer uso de los materiales off line 0 descargables y de esta manera dar un soporte al proceso de enseñanza-aprendizaje (Moll, 2016).

El uso del e-learning, dentro del ámbito educativo se asocia a diferentes cuestiones como son: educación superior, formación de personas adultas, formación ocupacional, entre otras, pero en los últimos años, éste se ha extendido hacia los diferentes niveles educativos (Mella, Narváez \& Buhring, 2016). Por ello, se considera la incorporación del e-learning en las aulas de la educación media superior. En este sentido, en la Escuela Preparatoria número 1, de la Universidad Autónoma del Estado de Hidalgo (UAEH), se aplicó una encuesta a los alumnos para identificar sus preferencias con relación al uso de la tecnología para llevar a cabo su proceso de aprendizaje.

\section{Marco conceptual}

El aprendizaje electrónico ha permitido romper con los límites geográficos y espacio-temporales, brindando la posibilidad de incorporar sus beneficios dentro del proceso de aprendizaje, además de que su auge ha generado la emergencia de diversos tipos de aprendizaje electrónico, como son: e-Learning, bLearning, m-Learning y u-Learning. Los cuales describiremos a continuación, con base en los planteamientos de Santiago Moll (2016 y 2016b).

\section{E-learning}

El término e-Learning o aprendizaje electrónico no es nuevo, ya que las personas lo conocen tal vez con otro nombre: como puede ser formación on-line, cursos virtuales, teleformación, formación a distancia, entre otros; pero lo que sí resulta novedoso es que este tipo de aprendizaje brinda grandes beneficios para el estudio independiente y para la comodidad de los estudiantes, ya que ofrece flexibilidad en la administración del tiempo y del espacio.

El aprendizaje electrónico (e-Learning), es la educación a distancia que utiliza medios electrónicos conectados a internet o no y un software, que son usados por un grupo de personas para interactuar entre sí. Como parte de las figuras que participan en este tipo de aprendizaje identificamos al facilitador, al tutor y a los estudiantes. Por tanto, el e-Learning puede ser definido como una modalidad de aprendizaje dentro de la Educación a
Distancia, en donde se utilizan las redes de datos como medios (Internet, Intranets, etc.), las herramientas o aplicaciones hipertextuales como soporte (Correo electrónico, web, Chat, etcétera) y los contenidos y/o las unidades como aprendizaje en línea.

Dentro de las ventajas que podemos encontrar, en este tipo de aprendizaje destacan las siguientes:

- Rapidez y agilidad en el proceso de búsqueda de información, ya que el usuario puede trabajar por medio del internet.

-Just-in-time teaching. El usuario se puede trabajar en su proceso de aprendizaje en el momento que se necesita.

-Just-for-me. Permite un proceso de enseñanza y aprendizaje personalizado debido al uso de la tecnología.

-Feedback o retroalimentación. Ofrece la posibilidad de que el facilitador haga comentarios respecto a su trabajo.

- Competencia digital, permite adquirir conocimiento y habilidades que permiten asociar diversas dimensiones del aprendizaje, utilizando un entorno virtual.

\section{B-Learning}

El b-Learning o Blended learning contempla la combinación entre la enseñanza presencial y la no presencial, en el que la característica principal es el uso de tecnología, es decir, es un aprendizaje mixto, ya que dentro del aula de clases la metodología es presencial y las actividades pueden ser complementadas en línea. Por tanto, con base en Moll (2016) y Pérula (2011) en esta modalidad encontramos las siguientes ventajas:

-Flexibilidad en el aprendizaje, en los que el docente puede hacer uso de los diferentes medios electrónicos para generar el conocimiento en los estudiantes.

-Papel determinante del docente, ya que la tecnología es vista como una herramienta para el aprendizaje y el docente se convierte en el facilitador dentro del proceso de enseñanza y aprendizaje.

-Aula como espacio para el aprendizaje y la cooperación. En este caso, el aula se convierte en el espacio donde se aprende a cooperar mediante el uso de diversas metodologías activas como el Aprendizaje Basado en Proyectos (ABP)

-Materiales digitales. Mediante el uso de diversos materiales digitales se permite el alojamiento del diseño de algunas actividades de enseñanza o de los productos de aprendizaje y logro en alguna plataforma de carácter educativo.

Dentro de este tipo de aprendizaje electrónico, podemos utilizar el modelo pedagógico denominado Flipped 
Classroom o también conocido como aula invertida o clase al revés. Tal como la define Raúl Santiago (2015), se identifica como un Modelo pedagógico que transfiere determinados procesos de enseñanza y aprendizaje fuera del aula y utiliza el tiempo de clase, juntamente con la experiencia docente, para facilitar y potenciar procesos de adquisición y práctica de conocimientos dentro del aula. Por lo que éste es un ejemplo muy claro de lo que es el e-Learning.

\section{M-Learning}

Actualmente algunos aprendizajes electrónicos están definidos por el uso de las tecnologías que requieren de internet, esto ha proliferado con el uso de dispositivos móviles, debido a las ventajas que ofrecen en comparación a otros medios electrónicos. Estos gadgets están brindando nuevas oportunidades de aprendizaje, desafiando las habilidades de los usuarios al integrar dinámicas que generan y favorecen nuevas experiencias de aprendizaje, por medio de aplicaciones o programas enfocados a algún tipo de conocimiento, que puede ser asimilado por medio de actividades o juegos enfocados a diversas áreas.

El $m$-Learning o mobile learning es un aprendizaje que se puede aplicar dentro y fuera del aula de clases. Esto debido al uso de los dispositivos móviles que tanto estudiantes como docentes tienen acceso, como con los Smartphone o la Tablet. Actualmente estos dispositivos permiten que cualquier miembro de la sociedad pueda acceder al conocimiento que se encuentra disponible gracias a internet, dado que la tecnología se ha hecho más accesible, a diferencia de hace algunos años, cuando la computadora era la única forma de entrar a la red.

Otra de las condiciones que apoya la accesibilidad es que los dispositivos móviles que existen en el mercado, son de diversos precios, lo cual permite que casi cualquier persona pueda acceder a este tipo de tecnología, de una manera práctica, eficiente y eficaz (Pérez y Delgado, 2018).

\section{U-Learning}

Ubiquitous learning o aprendizaje ubicuo. En este tipo de aprendizaje se suman los tres aprendizajes anteriores, ya que éste considera la noción de que la incorporación del conocimiento se puede realizar mientras se llevan a cabo diversas actividades paralelas en los diferentes espacios que se utilicen, por lo que el aprendizaje se puede entender como una actividad que puede ejercerse en cualquier momento y en cualquier lugar (Moll, 2016).

El u-Learnign permite generar un verdadero aprendizaje autónomo, utilizando las ventajas que ofrecen las tecnologías de comunicaciones. Este tipo de aprendizaje es resultado de la unión de diversas tecnologías con diferentes tipos de aprendizaje como son: m-Learning, bLearning, web 2.0, entre otras, las cuales hacen uso de las tecnologías actuales. Esta modalidad permite aprender en cualquier momento y cualquier lugar, dado que los recursos se encuentran al alcance del usuario, con solo tener un dispositivo electrónico y una conexión a internet.

\section{Uso de los dispositivos móviles dentro del aula}

Uno de los retos que enfrentamos los docentes, ante los cambios tecnológicos y los estilos de aprendizaje que se generan día tras día, es la adaptación a estas nuevas realidades, donde el aprendizaje ya no sólo se concentra en el aula, sino que debemos considerar el uso de las TIC en los procesos pedagógicos. Pero además, somos conscientes de que los alumnos utilizan los diferentes dispositivos móviles tanto en la vida cotidiana como en el ámbito académico. Así que consideramos que se puede aprovechar el uso de estos dispositivos dentro del proceso de enseñanza- aprendizaje de la siguiente manera:

- Como herramienta de investigación. Permite el uso de la red para la consulta de diferentes materiales, como son: libros, presentaciones, artículos, tutoriales, entre otros. Lo que facilita tener la información en línea.

- Como herramienta de comunicación e interacción entre los diferentes actores. La interacción y comunicación puede darse con los docentes y alumnos, sean internos y externos, además se puede desarrollar dentro o fuera del aula, para la aplicación de actividades como juegos, autoevaluaciones, entre otras actividades que determinen los usuarios.

- Como herramienta de creación y gestión de los diversos contenidos didácticos que apoyan a la enseñanza y al aprendizaje, así como la administración de fechasclaves, tareas, materiales didácticos (imágenes, videos, audios, podcast, etc.).

- Como herramienta de colaboración entre los diferentes actores, la cual se puede generar de manera síncrona o asíncrona. Un ejemplo de esto puede ser la producción de textos o presentaciones construidas de manera colaborativa en línea.

- Como herramienta para la individualización, permite la generación de actividades en las que se contemplen los estilos de aprendizaje y el nivel académico de cada uno de los alumnos o bien de un determinado grupo de alumnos. 


\section{Resultados}

Los diferentes dispositivos electrónicos, y en específico el teléfono inteligente, han generado cambios en las comunicaciones en la sociedad, así los estudiantes en estos contextos buscan la innovación en las formas de la enseñanza, ya sea revisando contenidos sobre la asignatura o realizando juegos que retroalimenten la información vista en el aula de clase.

Por ejemplo, las formas de comunicación oral y escrita van cambiando por la velocidad y programación de códigos en el teléfono inteligente (Pérez y Delgado, 2018). Por ello, la inclusión de las tecnologías en las estrategias del docente ha sido ampliamente reconocida como fuente de motivación para los estudiantes.

Con el fin de recabar información específica sobre la opiniones que tienen algunos estudiantes al respecto, se realizó una encuesta sobre uso de medios electrónicos como estrategia de enseñanza desde una perspectiva de aprendizaje dinámico e interactivo, la cual tuvo como objetivo contar con información sobre las preferencias que tienen los estudiantes de bachillerato para identificar las características que los medios de enseñanza deben tener para que les resulten atractivos.

La población objetivo fueron los estudiantes que cursan el segundo semestre en el periodo enero-junio, 2019, en la Escuela Preparatoria Número Uno que pertenece a Universidad Autónoma del Estado de Hidalgo.

El propósito del levantamiento de esta encuesta es contar información sobre el uso que dan los jóvenes de preparatoria a los medios electrónicos, para con base en ello diseñar estrategias para generar aprendizajes dinámicos e interactivos

La encuesta fue aplicada a una muestra de 265 estudiantes con una fiabilidad de 0.89 obtenida con la prueba Alfa de Cronbach, realizada en el programa SPSS.

De la muestra analizada, $58 \%$ de estudiantes pertenecen al género femenino y $42 \%$ al masculino, con edad promedio de 15 años, 3 meses para las mujeres y 15 años, 5 meses para los hombres.

Las preguntas realizadas permiten conocer la opinión de los estudiantes sobre el uso de las tecnologías en su día a día escolar. Para lo que tomaremos en cuenta que cada pregunta arroja un resultado importante, que será considerado para el diseño de estrategias didácticas, las cuales se podrán aplicar a los planes de estudio que actualmente se están impartiendo en la Escuela Preparatoria Número Uno.
Por cuestiones de espacio, para este artículo sólo se analizan algunas preguntas, que son las más relevantes y representativas de los datos para esta investigación.

La primera pregunta tiene relación con la percepción de los estudiantes sobre el uso de medios electrónicos en los espacios escolares, encontrando que las respuestas registradas, presentan una notable tendencia a encontrar las clases más dinámicas y divertidas cuando el docente emplea algún dispositivo electrónico, para impartir sus clases, como tal como se muestra en la figura 1 , donde la mayoría $59 \%$ de los encuestados está totalmente de acuerdo con este uso.

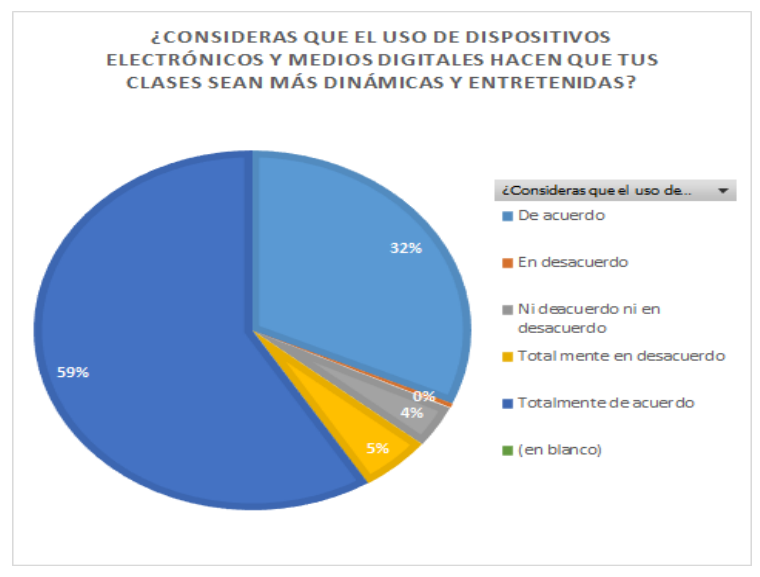

Figura 1: Gráfica de la pregunta 1.

En cuanto a la segunda pregunta, refiere al gusto por el uso de los materiales electrónicos y la tendencia también apunta al gusto por desarrollar sus actividades por medio de dispositivos electrónicos, ya que $54 \%$ está totalmente de acuerdo con ello.

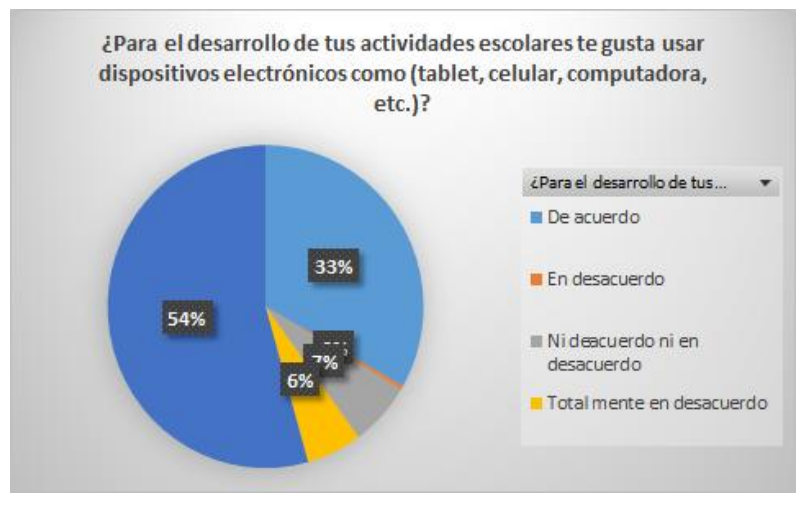

Figura 2: Gráfica de la pregunta 2.

En cuanto al uso del celular para actividades escolares una gran mayoría (79\%) está a favor de su uso en dichas actividades, tal como se muestra en la figura 3. 


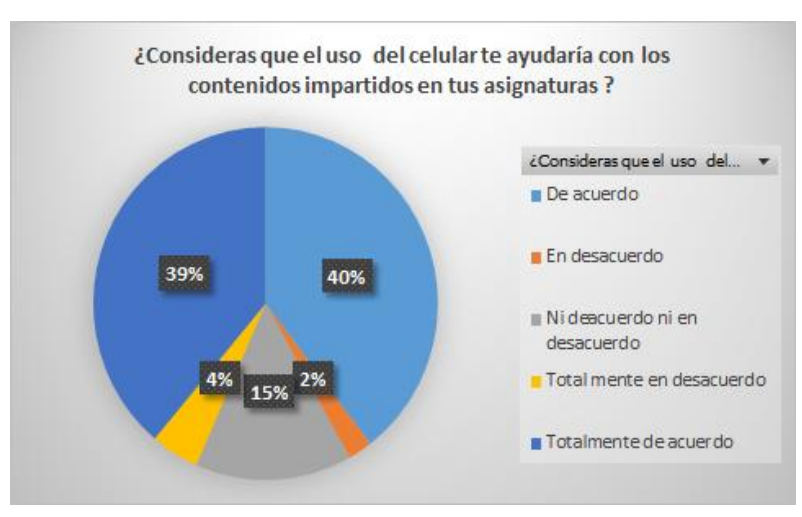

Figura 3: Gráfica de la pregunta 3.

Al considerar los resultados de estas preguntas, se puede observar que existe una tendencia al uso de las tecnologías de la información como herramientas de aprendizaje. En este caso, el celular es de suma importancia para los estudiantes, pues de acuerdo con la respuesta a la pregunta 3 , es notable el $78.49 \%$ de aprobación, por lo que ellos prefieren utilizar este gadget.

El uso de la tecnología móvil en el aula es un tema que genera diversos debates entre los docentes y directivos de las instituciones educativas. Por ello, es importante ver a los dispositivos móviles como herramientas, más que como enemigos de la educación, dándoles un valor similar al que damos a las computadoras, los libros o los manuales.

Como cualquier herramienta, los dispositivos móviles tienen aspectos positivos y negativos, los cuales son acordes con las necesidades del estudiantado, el profesorado, los objetivos de aprendizaje, y las actividades a realizar, entre otras.

La importancia de utilizar los dispositivos móviles dentro del aula radica en las ventajas didácticas y pedagógicas que se pueden tener, ya que de acuerdo con los resultados de la encuesta realizada a estudiantes del segundo semestre de nivel medio superior, se muestra mayor aceptación y motivación por el aprendizaje mediante el uso de los dispositivos móviles, y al mismo tiempo, su uso permite que los estudiantes trabajen de manera colaborativa y cooperativa, al establecer comunicación permanente en y para el desarrollo de sus trabajos, lo que también ofrece posibilidades para el desarrollo del pensamiento crítico.

Dentro del aula se puede aprovechar el uso de los teléfonos móviles o Smartphone, ya que de acuerdo con los referentes manejados en el marco conceptual (INSPIRATICS, S/F; Mella, Narváez y Buhring, 2016) son herramientas de apoyo para la búsqueda de información en todo momento, puesto que permiten a los estudiantes tener acceso a internet en todo momento, generando flexibilidad en la realización de actividades o tareas, (participar en un blog, aprender a buscar, y publicar contenido) acceder a la información (libros, periódicos, revistas) y esto se puede lograr estableciendo reglas de uso en el espacio escolar, lo que permitirá aprender a utilizar la tecnología de una manera adecuada y con responsabilidad.

También se puede trabajar con realidad aumentada, código $\mathrm{QR}$, quizz o redes sociales, fomentando en los estudiantes el interés por la participación y la motivación para el desarrollo de todo tipo de actividades para su aprendizaje; asimismo, al docente le permite identificar metodologías personalizadas según las necesidades del estudiantado y al mismo, tener tiempo y oportunidad para dar seguimiento (De Benito, 2000; Granić, Ćukušić y Walker, 2009).

\section{Conclusiones}

Actualmente, la escuela debe ser capaz de adaptarse a los diferentes cambios tecnológicos, por ello, consideramos que la incorporación de los cuatro aprendizajes electrónicos dentro del aula, combinados con diversas metodologías activas, que permitirán el fomento a la cooperación, la inclusión y, por supuesto, la solución de problemas que se tienen dentro de una enseñanza tradicional, ofrecen posibilidades para el enriquecimiento de los aprendizajes.

Mediante la aplicación de la encuesta, denominada "Uso de medios electrónicos como estrategia de enseñanza para generar un aprendizaje dinámico e interactivo", aplicada a los estudiantes del segundo semestre de la Escuela Preparatoria Número Uno, se identificó que el uso de los diferentes tipos de aprendizaje electrónico les permite llevar a cabo un proceso de enseñanza aprendizaje más efectivo, consiguiendo con ello aprendizajes significativos.

Por lo que, el uso del m-Learning junto con el u-Learning, se pueden aprovechar para aumentar el rendimiento de los estudiantes, ya que en ocasiones el tiempo que se tiene para el desarrollo de una clase presencial se ve afectado por diversos factores, que impiden que se dé un aprovechamiento adecuado, generando dudas y un bajo rendimiento que se puede ver reflejado en la deserción escolar.

Con base en los planteamientos anteriores, se generan diversas reflexiones que conllevan a realizar una reestructuración del proceso de enseñanza y aprendizaje. Utilizar, en particular, el m-Learning y el uLearning en la creación de cursos y/o actividades, donde se utilicen las diferentes tecnologías con las que contamos hoy en día, ofrece una amplia gama de posibilidades, ya que se pone en manifiesto que el uso 
de los Smartphone y tabletas permiten obtener mayor grado de aceptación por parte de los estudiantes y de esta manera no se encuentran tan dispersos, y al mismo tiempo, con el uso de este tipo de aprendizaje podemos atender los diferentes estilos de aprendizaje.

De igual manera, se puede observar la aceptación por parte del estudiantado en el uso del celular para la realización de actividades académicas, debido a que este tipo de dispositivo tiene acceso a internet, herramienta que les permite consultar información, ingresar a diversas plataformas educativas, tener acceso a recursos didácticos que son proporcionados por los docentes o la institución, como lo es la plataforma educativa o la biblioteca digital, por poner un ejemplo, así como mantenerse informados con relación a alguna temática particular que surja en el desarrollo de la clase presencial. Cabe señalar, que al mismo tiempo se requiere una orientación para que los estudiantes puedan seleccionar la información válida y veraz, esta orientación debe ser proporcionada por el docente como facilitador del aprendizaje y juega un papel importante dentro de este proceso.

El gran reto que tenemos como docentes, es adaptarnos a una nueva realidad, en donde el aprendizaje ya no se concentra solo en el aula, sino que se debe considerar el uso de las Tecnologías de la información y la comunicación, y de esa forma, contar con un espacio virtual que nos ayude a equilibrar la enseñanza semipresencial y la enseñanza a distancia.

En la mayoría de las instituciones o centros educativos, tanto los directivos como los docentes optan por prohibir los dispositivos móviles dentro del aula, comentando que son un distractor para los alumnos (INSPIRATICS, S.F.), pero lo cierto es que es algo que choca con nuestra realidad, ya que cada vez es más común el uso de estos aparatos, por lo que podemos aprovechar las ventajas que se puedan tener y usarlos con fines educativos y al mismo generar el valor de la responsabilidad en cuanto al uso de las tecnologías.

Así que podemos utilizarlos como directrices para el aprendizaje móvil, al usar todas las aplicaciones básicas que contiene un Smartphone como la cámara de video o de fotografías, la agenda, tabla de notas, editor de imágenes, entre otras aplicaciones; todo esto permitirá facilitar el aprendizaje, dando respuesta a la evaluación de manera inmediata y una retroalimentación adecuada por parte del docente.

Anexo:

Nota: Este anexo se presenta con la finalidad de que el lector tenga mayor claridad sobre el tipo de información que se obtuvo y cómo es que a partir de ella se pueden realizar ciertas afirmaciones. De la misma manera es importante mencionar que todas las respuestas fueron obtenidas mediante escala Licker que ofrece las siguientes cinco opciones, por lo que no se incluyen aquí después de cada pregunta. 1) Totalmente en desacuerdo 2) En desacuerdo 3) Ni de acuerdo ni en desacuerdo 4) De acuerdo 5) Totalmente de acuerdo

\section{Encuesta aplicada}

Uso de medios electrónicos como estrategia de enseñanza para generar un aprendizaje dinámico e interactivo.

Objetivo: Analizar las características que los medios de enseñanza deben poseer para que resulten atractivos para los estudiantes.

Instrucciones: Responde las siguientes preguntas de acuerdo a tu criterio.

\section{Edad: $\quad$ Género: Femenino / Masculino}

1. ¿Consideras que el uso de dispositivos electrónicos y medios digitales hacen que tus clases sean más dinámicas y entretenidas?

2. ¿Para el desarrollo de tus actividades escolares te gusta usar dispositivos electrónicos como (tablet, celular, computadora, etc.)?

3. ¿Consideras que el uso del celular te ayudaría con los contenidos impartidos en tus asignaturas?

4. ¿Para el desarrollo de tus clases el uso de la computadora es necesario?

5. ¿Te gustaría usar la tableta electrónica como herramienta en tus materias?

6. ¿Aprendes con mayor facilidad utilizando materiales que se encuentran en internet de forma autónoma, sin la supervisión de un profesor?

7. ¿La idea de aprender utilizando aplicaciones móviles en tu celular te resulta atractiva y divertida?

8. ¿En tus clases te agradaría utilizar kahoot, quizz, duolingo, entre otros para reafirmar tus conocimientos?

9. ¿Para revisar el contenido de tus asignaturas utilizas las diferentes plataformas educativas (Schoology, edmodo, plataforma garza, etc.)?

10. Te agrada leer un libro de manera electrónica, utilizando una tablet o un Smartphone. 


\section{Referencias bibliográficas}

Benito-Osorio, Diana. “Aprendizaje en el entorno del e-learning: estrategias y figura del moderador". Revista de Universidad y Sociedad del Conocimiento, 2009.

De Benito, B (2000). Herramientas para la creación, distribución y gestión de cursos a través de Internet. Edutec. Revista Electrónica de Tecnología Educativa, $12 . \quad$ Disponible http://edutec.rediris.es/Revelec2/Revelec12/PDF/deBenito.pdf consultado: 28 febrero, 2019.

DocTutor. 2015. http://www.doctutor.es/2011/12/02/ideas-y-reflexiones-eneducacion-medica-diciembre-2011/ consultado: 15 enero, 2019).

Granić, A., Ćukušić, M., \& Walker, R. mLearning in a Europe-wide network of schools. Educational Media International, 2009: 167-184.

INSPIRATICS. “EEl móvil en el aula? Ideas, ventajas, retos y posibilidades”. (sin fecha), disponible en: https://www.inspiratics.org/es/recursoseducativos/el-movil-en-elaula-ideas-ventajas-retos-y-posibilidades (consultado: 20 de febrero, 2019).

Mella, P. O., Narváez, C. G., \& Buhring Bonacich, K. "Valoración del mlearning en el proceso de aprendizaje de estudiantes de la Salud". Revista Cubana de Educación Media Superior, 2016: 372-381. Disponible en: http://search.ebscohost.com/login.aspx?direct=true \&db=a9h\&AN=12 2959261\&site=ehost-live consultado: 24 de febrero, 2019.

Moll, Santiago. "Tipos de aprendizajes electrónicos para aplicar dentro y fuera del aula”. Educación 3.0. Disponible en https://www.educaciontrespuntocero.com/noticias/tiposdeaprendizajeselectronicos/97794.html?fbclid=IwAR16 obqGivRqy VsIr3PcuSvW_j_h9okzaCEHh_Qo3N332etkA0Hy4nyDA consultado: 01 enero, 2019.

Moll, Santiago. "Tres tipos de aprendizaje electrónicos para la escuela del siglo XXI”. 2016. Disponible en: https://www.mesaticfid.cl/tres-tipos-deaprendizaje-electronicos-para-la-escuela-del-siglo-xxi/ consultado: 22 de febrero, 2019).

Pérez, M., \& Delgado, A. "Medios Móviles Emergentes en la Enseñanza de Lenguas”, pp.114-128. Revista de Investigación Social. Prisma Social $\mathrm{N}^{\circ} 20$, marzo 2018.

Pérula de Torres, Luis A. "El aprendizaje electrónico (e-learning, b-learning)". (2 de diciembre, 2011). Disponible en: https://www.doctutor.es/.../ideas-y-reflexiones-en-educacion-medicadiciembre-2011/ consultado: 15 enero, 2019).

Santiago, Raúl. (2015) "9 Maneras de utilizar Dispositivos Móviles en el aula”. The Flipped Classroom. 2015. Disponible en: https://www.theflippedclassroom.es/9-maneras-de-utilizardispositivos-moviles-en-el-aula/ consultado: 18 febrero, 2019. 